Copyright by the Acoustical Society of America. Gentry, C. A., Guigou, C., \& Fuller, C. R. (1997). Smart foam for applications in passive-act radiation control. Journal of the Acoustical Society of America, 101(4), 1771-1778. doi: 10.1121/1.418234

\title{
Smart foam for applications in passive-active noise radiation control
}

\author{
C. A. Gentry, C. Guigou, and C. R. Fuller \\ Vibration and Acoustics Laboratories, Virginia Polytechnic Institute and State University, Mechanical \\ Engineering Department, Blacksburg, Virginia 24061-0238
}

(Received 19 April 1996; revised 8 October 1996; accepted 25 November 1996)

\begin{abstract}
The development of a smart foam for passive-active noise control is discussed. The smart foam consists of cylindrically curved sections of PVDF film embedded in partially reticulated polyurethane acoustic foam forming a very thin, compact arrangement. The device is designed to reduce sound by the simultaneous action of the passive absorption of the foam (effective at high frequencies) and the active input of the PVDF element driven by an oscillating electrical input (effective at low frequencies). The PVDF actuator is configured to behave in a linear sense and to couple in-plane strain associated with piezoelectric effect with out-of-plane motion needed to radiate sound from the foam surface. The performance of the passive-active device is studied for controlling sound radiation from a vibrating piston. A feedforward LMS controller is used to minimize the signal from a far-field error microphone. Results of harmonic and broadband control are presented. The potential of the smart foam for globally reducing sound radiation in the low- and high-frequency range is demonstrated. (C) 1997 Acoustical Society of America.
\end{abstract}

[S0001-4966(97)03704-1]

PACS numbers: 43.10.Ln, 43.50.Gf, 43.50.Ki [PJR]

\section{INTRODUCTION}

In the last few years, there has been increased interest in the reduction of sound and/or vibrations by use of hybrid active-passive control techniques. There are two general types of active-passive noise control approaches: the adaptive-passive techniques and active-passive hybrid techniques. The adaptive-passive techniques are essentially passive devices whose static properties are adapted or changed to optimize their performance. ${ }^{1}$

An example of an adaptive-passive control system uses electro-rheological fluids (ER), which undergo significant changes in their mechanical properties, such as their mass distribution, and energy dissipation characteristics, when subjected to electric fields. These fluids can be embedded in voids in structural materials enabling the global static properties (i.e., damping, stiffness, etc.) of the structure to be controlled. ${ }^{2}$ Another adaptive-passive method utilizes adaptively tuned vibration absorbers ${ }^{3}$ (whose simple form is a spring-mass system), which are being studied for aircraft applications to limit fuselage response to engines and propeller excitations. ${ }^{4}$

Active-passive hybrids use both active and passive elements in either series or parallel. The passive device usually carries the primary vibration attenuation characteristic, while the active component is used to enhance the performance of the passive system or overcome the limitations of the passive system. The active-passive approach is used in this paper to develop an active smart foam. The smart foam design integrates a distributed piezoelectric polymer actuator (the active component) between individual layers of sound-absorbing foam (the passive component). Smart foam can be bonded directly to a vibrating structure and act as an active surface coating, which provides reduction of structural sound radiation. Further details concerning active-passive surface coat- ings will be given but first a brief overview of passive sound reduction techniques is needed.

Porous sound-absorbing materials are commonly implemented for a large range of applications (such as buildings, machinery enclosures and aircraft) to reduce sound propagation. The use of a polyimide foam layer has been investigated for reducing sound transmission. ${ }^{5}$ In noise control applications, which employ passive sound absorbers, the air molecules in the interstices of the porous material oscillate with the frequency of the exciting sound wave. This oscillation results in frictional losses. Changes in flow direction and expansions and contractions of the flow throughout irregular pores result in a loss of momentum in the direction of wave propagation. These two phenomena account for most of the energy losses in the high-frequency range. ${ }^{6}$ However, a porous layer can absorb a large amount of acoustic energy only if its thickness is comparable to the wavelength of the incident sound. This implies that such a passive technique is not very efficient in the low-frequency region.

Active control techniques can be used to enhance the sound reduction of passive sound absorbing materials in the low frequencies. Bolton et al. ${ }^{7}$ presented analytical results demonstrating that the low-frequency performance of a finite-depth layer of elastic porous material may be enhanced by applying an appropriate force to the solid phase at the front surface of the layer. They showed that at any angle of incidence, the solid phase of the foam may be forced so as to create a perfect impedance match with the incident plane wave, thus causing the sound to be completely absorbed. However, no physical implementation of such a device was discussed. An active-passive piezocomposite actuator has been experimentally developed and tested for underwater sound reduction. ${ }^{8}$ The design of the active coating prevents an incident sound wave from reflecting off the acoustic 
boundary of an object submerged in a heavy fluid. The acoustic energy associated with the incident disturbance is absorbed out of the medium through a piezocomposite device in the coating and dissipated by internal electrical means. This elastomer coating consists of a piezoelectric actuator and piezoelectric sensors embedded in an elastomer and controlled by an analog feedback network. The actuator was tested in a water-filled pulse tube and echo reduction levels were measured over an extended frequency band for tonal excitations. Good reduction was achieved and results clearly indicate how the active component of the device overcomes the limitations of a purely passive elastic coating. The results also indicate that by optimizing the embedded sensor location one can tune the piezocomposite coating to yield maximum echo reduction in a certain frequency band.

Another example of an active surface coating is actively controlled constrained layer damping (ACLD), which is an active-passive hybrid composed of a visco-elastic damping material sandwiched between two piezoelectric layers. ${ }^{9}$ The three layer composite ACLD when bonded to a vibrating surface acts as a smart constraining layer damping treatment with built in sensing and actuation capabilities. The sensing is provided by the piezoelectric layer, which is directly bonded to the vibrating surface. The actuation is provided by the other piezoelectric layer driven by a control voltage. With appropriate strain control, the shear deformation of the visco-elastic damping layer can be increased, the energy dissipation mechanism can be enhanced, and the structural vibration damping ratio is increased. Analytical results involving beam vibration control show the ACLD treatment is superior to conventional active control damping, particularly in the low-frequency range (first three vibration modes of the beam). Recently, a smart foam to be used as an activepassive sound absorber has been implemented by incorporating a piezoelectric polymer PVDF (polyvinylidene fluoride) film within a foam layer. ${ }^{10}$ This work provided the basis of the present study. The smart foam differs from previous work on sound reduction coatings in that it is designed to be used in air and incorporates a PVDF film embedded in a blown polyurethane foam. These materials are chosen because their impedance is closer to that of air when compared to the materials used to construct active surface coatings in underwater sound reduction applications. The PVDF film thus acts as an acoustic actuator to enhance the lowfrequency sound absorption of the foam.

The concept of the smart foam is to combine active and passive control strategies such that the sound attenuation can be efficiently achieved over a broad range of frequencies. In this paper, the smart foam noise suppression device, combining a lightweight, distributed active layer and a passive acoustic damping material, is developed for the purpose of minimizing structural acoustic radiation. A schematic of the smart foam is shown in Fig. 1. Such a device consists of a layer of cylindrically curved piezoelectric polymer PVDF film embedded in partially reticulated polyurethane foam. As mentioned previously, it is usually impractical to rely on a purely passive sound absorption system in the low-frequency range. Therefore, the PVDF element is incorporated and serves as the active input to contribute to the low-frequency

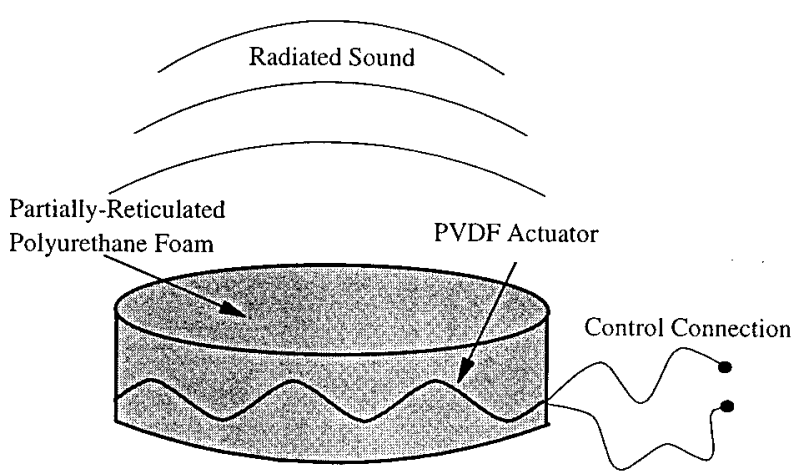

FIG. 1. Smart foam.

sound attenuation. The smart foam device differs from the ASAC control methods (see Ref. 11 for a discussion of ASAC) in that it adaptively modifies the acoustic impedance of a vibrating surface without the use of secondary control inputs directly mounted on the structure. It is also significantly different from common ANC techniques that use secondary acoustic sources (loud speakers) in arrays around the primary noise source because the smart foam is actually located on the surface of the vibrating structure. The goal of implementing smart foam is to modify the acoustic radiation impedance in order to yield a net decrease in the far-field sound power radiated by the vibrating noise source. While the most important advantage of implementing such a hybrid device in noise control applications is improved lowfrequency performance, other positive attributes of activepassive noise control devices are increased robustness, increased stability, and decreased control spillover. It should also be mentioned that the passive-active device proposed in this paper is relatively lightweight, which makes it a good candidate for aircraft noise control applications.

In this paper, the construction of the smart foam is first described. The PVDF actuator is cylindrically curved to couple the in-plane strain associated with the piezoelectric effect with the out-of-plane motion needed to radiate sound from the foam surface. The actuator is then configured such that nonlinear effects associated with the film curvature are minimized. The PVDF active layer is bonded between the two foam halves forming a very thin, compact arrangement. The performance of the active-passive device is then demonstrated for controlling sound radiation from a vibrating piston. The problem of suppressing piston sound radiation is addressed because it represents a simple source and generates a monopolelike radiation pattern. A successful solution to this simple noise control problem using smart foam has two advantages. It will provide insight into the fundamental method of operation of smart foam and represent an important step in the progression toward implementing smart foam in more complex noise control problems. One can interpret the vibration pattern of complex structures as a finite sum of multiple monopole sources. Therefore, an array of smart foam can be employed to attenuate the sound generated by a complex structural noise source. A feedforward LMS controller ${ }^{12}$ is used to minimize the signal from a far-field error microphone. Results of harmonic and broadband con- 
trol are presented. The potential of the smart foam for globally reducing sound radiation in the low- and highfrequency range is demonstrated.

\section{SMART FOAM CONSTRUCTION}

A sound-absorbing material, known as partially reticulated polyurethane foam, provides the passive element of smart foam and it dissipates incident acoustic wave energy through friction associated with the coupling of the liquid and solid phases of the foam. Partially reticulated polyurethane foam is an acoustical grade, open cell, flexible ester based urethane foam designed to give maximum sound absorption per given thickness. ${ }^{13}$ Since passive sound control is only significant at high frequencies, a PVDF film is embedded in the foam to implement the active control, which is most effective at low frequencies. Note that the actuator forces both the fluid and solid phases of the acoustic foam and acts in a speakerlike fashion. The main physical characteristic of the PVDF actuator is that it is intentionally curved to couple the predominantly in-plane strain associated with the piezoelectric effect and the out-of-plane motion, which is required to accelerate fluid particles and hence radiate sound away from the surface of the foam. A simple analytical model for a curved PVDF actuator revealed that, for a given input voltage, as the PVDF actuator (of constant dimensions) is changed from a flat configuration (infinite radius of curvature) to a half-cylinder configuration, the resulting out-ofplane displacement is increased. Since an increase of out-ofplane displacement yields an increase of sound radiation, a PVDF actuator curved into a series of half-cylinders was chosen for the smart foam design.

In a preliminary experiment, the smart foam included a continuous, homogeneous layer of $28-\mu \mathrm{m} \mathrm{Ag}$ metallized PVDF, which was curved into a series of four half-cylinders as shown in Fig. 2(a). Silver-electroded PVDF film was chosen as it can sustain high voltage amplitudes required for actuator applications. An electrical voltage of magnitude 30 $\mathrm{V} / \mu \mathrm{m}$ can be then applied to the PVDF actuator without electrical breakdown. Driving the actuator with an ac voltage across the top and bottom electrode [without changing polarity, as shown in Fig. 2(a)] resulted in poor radiation efficiency of the active-passive device. When electrically excited, cell 1 and 3 experienced a positive upward displacement. Conversely, cell 2 and 4 experienced a negative downward displacement. The net result is an inefficient sound source at low frequencies with dipole-type radiation characteristics. In addition, the corresponding asymmetry of operation under positive and negative voltage caused asymmetry in the mechanical coupling between the out-of-plane displacement and the strain in the uni-axial direction of the film. This nonlinear dynamic behavior appeared in the form of higher-order harmonic distortion in the acoustic frequency response spectrum.

These undesirable characteristics were overcome using an arrangement first suggested by Tibbets. ${ }^{14}$ Two improved PVDF actuator configurations are shown in Fig. 2(b) and (c). Each actuator is configured by dividing the continuous layer of PVDF film into several individual transducers by a chemical etching process. The etching process involves chemically (a)

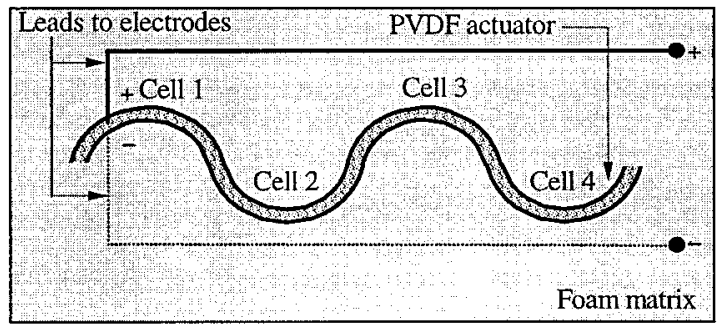

(b)

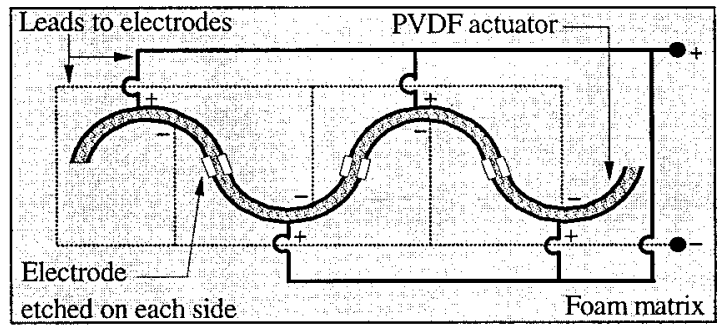

(c)

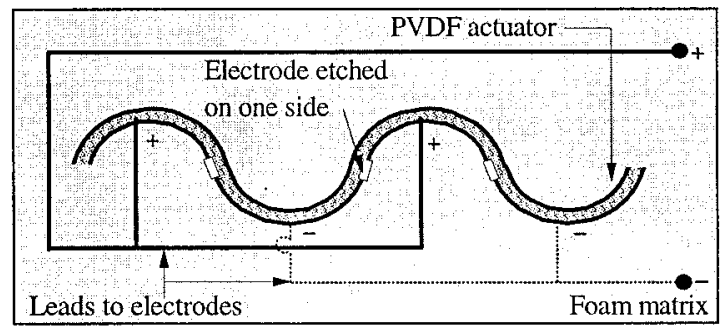

FIG. 2. PVDF actuator configuration. (a) Original; (b) parallel; (c) seriesparallel.

removing or erasing thin portions of PVDF film electrodes at the boundaries where the physical polarity (curvature) of the actuator changes. The actuator is then composed of several individual transducers, which can be driven with different voltages (same amplitude with $180^{\circ}$ phase difference). In the parallel actuator arrangement, as presented in Fig. 2(b), the top and bottom electrodes are then connected in parallel with phase reversal. Therefore, the PVDF film is activated such that neighboring cells are driven by the same voltage amplitude with an $180^{\circ}$ phase difference. The resulting effect is that each cell moves in the same out-of-plane direction yielding a net volumetric source strength and as a consequence an increase in sound radiation as compared to the preliminary PVDF configuration illustrated in Fig. 2(a). The seriesparallel actuator configuration, illustrated in Fig. 2(c), requires that only the bottom electrode is divided into independent transducers, which are then wired $180^{\circ}$ out of phase. This arrangement also results in a more efficient radiator as compared to the original configuration as the PVDF film is moving as a whole in the out-of-plane direction. A detailed comparison of the effectiveness of these improved PVDF actuators as sound radiators will be discussed in a subsequent section of this paper. Note that the periphery of the PVDF film actuator was etched as well to prevent arcing effects. Electrical leads were attached to the PVDF actuator with a copper foil adhesive tape reinforced with silver epoxy glue. To complete the construction process of the active-passive control device, the PVDF element was embedded in a layer of polyurethane foam. Note that the piston used in performance testing of smart foam was $15 \mathrm{~cm}$ in diameter and 


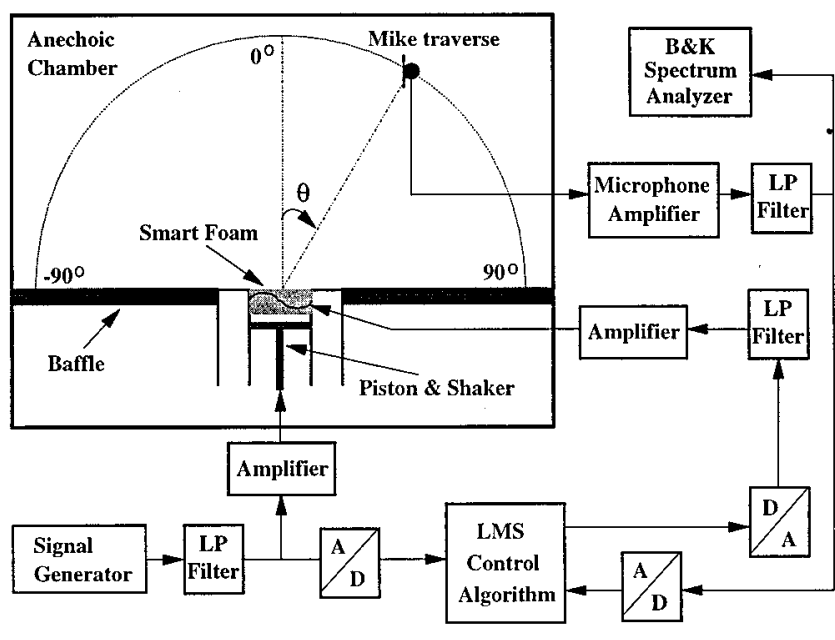

FIG. 3. Radiation control experimental setup.

dictated the size of the smart foam element to be fabricated. A series of four half-cylinder cells measuring approximately $2 \mathrm{~cm}$ in radius were cut through the cross section of a slab of foam measuring $15 \mathrm{~cm}$ in diameter and $5 \mathrm{~cm}$ thick. From a simple analytical model for the curved PVDF actuator, it was obtained that the largest radius possible for the PVDF actuator is optimal in terms of inducing out-of-plane displacement. Therefore, a $2 \mathrm{~cm}$ radius is used for each cell as it represents the largest that can be embedded within $5 \mathrm{~cm}$ of foam while allowing a minimum of $\frac{1}{2} \mathrm{~cm}$ of foam on the top and bottom surface of the device for protection of the piezoelectric layer. The PVDF actuator was bonded between the two circular foam halves retaining its curved shape and yielding a smart foam similar to that illustrated in Fig. 1. Silicon adhesive was chosen to bond the PVDF actuator because it is readily available and remains flexible once dry.

\section{EXPERIMENTAL SETUP AND PROCEDURE}

The radiation control experimental setup is shown in Fig. 3. The smart foam was manufactured as described in the previous section, and positioned on the surface of an oscillating piston mounted in a rigid baffle inside an anechoic chamber. The piston excitation was produced by a shaker driven by an external signal generator. A feedforward filtered-x LMS algorithm ${ }^{12}$ was used to determine the appropriate control signal necessary to minimize the radiated sound at an error microphone. This error microphone was located in the far field at $1.5 \mathrm{~m}$ in the perpendicular direction from the device surface (direction $\theta=0^{\circ}$ in Fig. 3). The control algorithm was implemented with a TMS320C30 DSP board resident in a personal computer. Note that the feedforward controller used the signal sent to the piston shaker as a reference signal (however, a signal from an accelerometer on the piston could also be used). A microphone located on a stepper motor driven traverse swept from $-90^{\circ}$ to $90^{\circ}$ at a radial distance of $1.5 \mathrm{~m}$ from the rigid baffle plane for 21 equally spaced measurement points. The far-field pressure levels were measured under three conditions:

(1) the bare piston operating alone (piston);
(2) the piston with smart foam and no control signal applied (passive control);

(3) the piston with smart foam and control signal applied (active-passive control).

The recorded sound-pressure levels (SPL) in $\mathrm{dB}$ relative to $20 \mu \mathrm{Pa}$ are presented for these three conditions to evaluate the efficiency of the passive-active device. A $20-\mu \mathrm{Pa}$ reference is used in all subsequent sound-pressure level calculations. The mechanical shaker used to drive the piston appeared to have two resonant frequencies at 290 and $472 \mathrm{~Hz}$ in the frequency band studied. Harmonic control was performed at 290 and $1000 \mathrm{~Hz}$, respectively. These frequencies were chosen to illustrate how the smart foam has the ability to successfully attenuate low- and high-frequency sound. Broadband control was also investigated and in this case, the piston was driven with band limited random noise 0-1600 Hz. Note that for broadband control, a 15-ms delay was added to the disturbance path to make the system more causal. This enabled the controller to adapt the coefficients necessary to compensate the nonminimum phase zeros of the control and error path. It should also be mentioned that, in the following discussion, the term "global" means "for all radiation angles $-90^{\circ} \leqslant \theta \leqslant 90^{\circ}$ " and "global sound level", refers to the average of the squared sound pressure measured at the 21 equally spaced microphone positions between $\theta=$ $-90^{\circ}$ to $\theta=90^{\circ}$ and converted to $\mathrm{dB}$ relative to $20 \mu \mathrm{Pa}$.

\section{COMPARISON OF VARIOUS ACTUATOR CONFIGURATIONS}

Linearization of the PVDF actuator dynamic response proved to be an important achievement in the smart foam development because it is associated with an increased radiation efficiency of the smart foam and therefore an increased sound reduction capability. To illustrate and compare the performance of the different actuator configuration referred to as the original PVDF actuator, the parallel actuator, and the series-parallel actuator, a simple experiment was performed in the anechoic chamber. The smart foam was positioned near the surface of the piston and in this case, was used as a noise source (i.e., the piston was stationary). For the different configurations, the PVDF actuator was driven at $290 \mathrm{~Hz}$ using an input voltage of 25, 50, 75, and $100 \mathrm{~V} \mathrm{rms.}$ Note that the maximum voltage allowed to drive the PVDF actuator is about $300 \mathrm{~V} \mathrm{rms}$. The far-field sound-pressure level (SPL) was measured at the driving frequency as well as at the first and second harmonic frequencies with a microphone positioned normal to the foam surface (at a radial distance of $1.5 \mathrm{~m}$ in the direction $\theta=0^{\circ}$ ) for each voltage increment.

Figure 4(a) presents the SPL for the three different actuator configurations using an input voltage of $100 \mathrm{~V} \mathrm{rms}$ at $290 \mathrm{~Hz}$. It can be seen that the series-parallel and parallel actuators are more than $15 \mathrm{~dB}$ louder than the original actuator. The parallel configuration yields an SPL about $6 \mathrm{~dB}$ higher than the series-parallel as it uses the both sides of PVDF electrode. The parallel actuator corresponds to the best configuration for increasing the SPL radiated by the smart foam. The linearity of the system with respect to the 
(a)

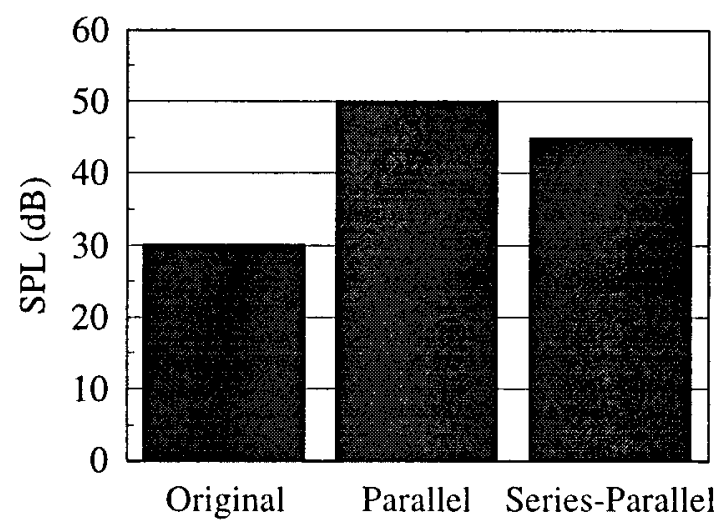

(b)

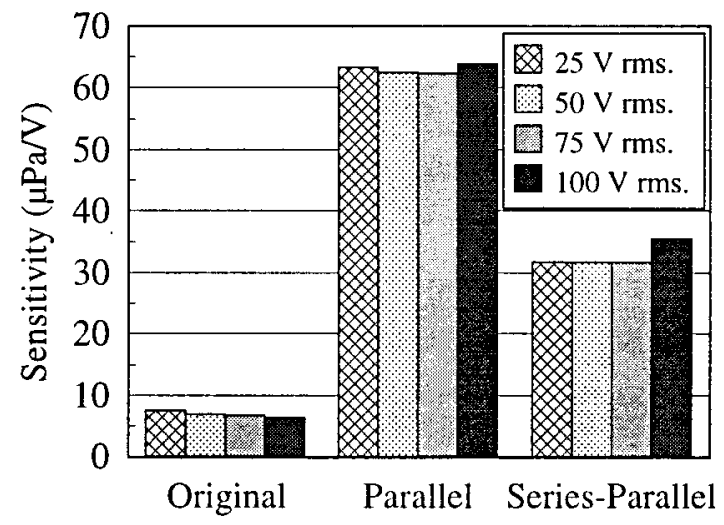

(c)

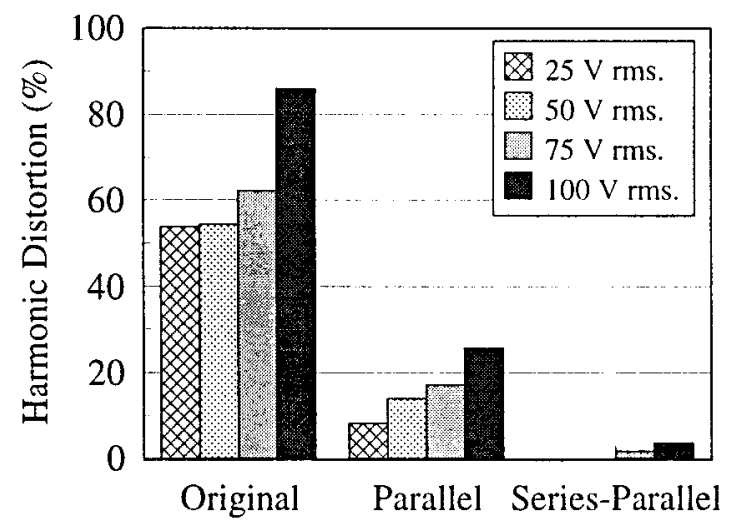

FIG. 4. Smart foam behavior at $290 \mathrm{~Hz}$ for different PVDF actuator configurations: (a) SPL under $100 \mathrm{~V}$ rms input voltage; (b) sensitivity; (c) harmonic distortion.

driving voltage or sensitivity is studied next. The sensitivity defined as the rms pressure divided by the input voltage is presented in Fig. 4(b). At the driving frequency, the sensitivity should ideally be a constant (as doubling the driving voltage is expected to double the radiated rms pressure). Indeed, the sensitivity is virtually constant for the three actuator con- figurations showing that the smart foam is linear with respect to the input voltage. It should also be noted that the sensitivity for the parallel configuration is the highest as it corresponds to the loudest system [see Fig. 4(a)] and is twice that of the series-parallel configuration. Finally, the nonlinear behavior in the form of harmonic distortion is investigated for the different actuator configurations. Harmonic distortion ${ }^{15}$ is a measure of the pressure amplitude distortion and refers to the deviation from correspondence between the acoustic output wave and the electrical input wave that results from nonlinear effects in the smart foam. It is defined as the ratio of the pressure amplitude of the harmonics to those of the fundamental and the harmonics, i.e.,

$$
\text { Distortion (in percent) }=100 \sqrt{\frac{p_{2}^{2}+p_{3}^{2}+p_{4}^{2}+\cdots}{p_{1}^{2}+p_{2}^{2}+p_{3}^{2}+\cdots}},
$$

where $p_{1}$ represents the pressure at fundamental frequency, $p_{2}$ the pressure at the second harmonic, and so on. It can be seen in Fig. 4(c) that the original actuator configuration is associated with large harmonic distortion (more than $85 \%$ when driven with $100 \mathrm{~V} \mathrm{rms}$ ) compared to the other two PVDF actuator configurations. It is also observed that the distortion level is increased as the input voltage to the PVDF actuator is increased. The series-parallel actuator corresponds to the best configuration for minimizing the harmonic distortion of the smart foam. Note that the harmonic distortion associated with the original PVDF actuator configuration was observed in the experiments presented in Ref. 10.

It can be deduced that the parallel and series-parallel actuator PVDF configurations greatly improve the acoustic response of the smart foam. Both PVDF arrangements yield increased sound output and much less harmonic distortion than the original PVDF configuration. It is also believed that the nonlinear characteristic (predominantly in the form of harmonic distortion) can be further decreased by using a much thinner bonding layer to embed the PVDF actuator within the foam (i.e., the use of spray glue instead of silicon adhesive is under investigation). Silicon adhesive has a high viscosity compared to spray glue adhesive. This high viscosity induces a shearing effect in the smart foam, which promotes a nonlinear coupling of the PVDF in-plane strain and the displacement in the normal direction.

\section{PASSIVE EFFECT OF SMART FOAM ON SOUND RADIATION}

In this section, the passive sound attenuation associated with the smart foam is studied and compared to that of a similar configuration of homogeneous plain acoustic foam. It is of interest to observe the effect of the PVDF actuator as well as the glue layer (used to bond the PVDF film to the foam) on the passive sound attenuation. Ideally, the passive sound control performance should not be reduced by the active layer embedded in the acoustic foam. A plain, homogeneous layer of acoustic foam (same dimensions as the smart foam) was used for comparison of passive performance. The piston was then driven by broadband random noise $(0-1600$ $\mathrm{Hz}$ ) and the global sound level (as previously defined) was obtained. Figure 5 presents the global sound level for the 


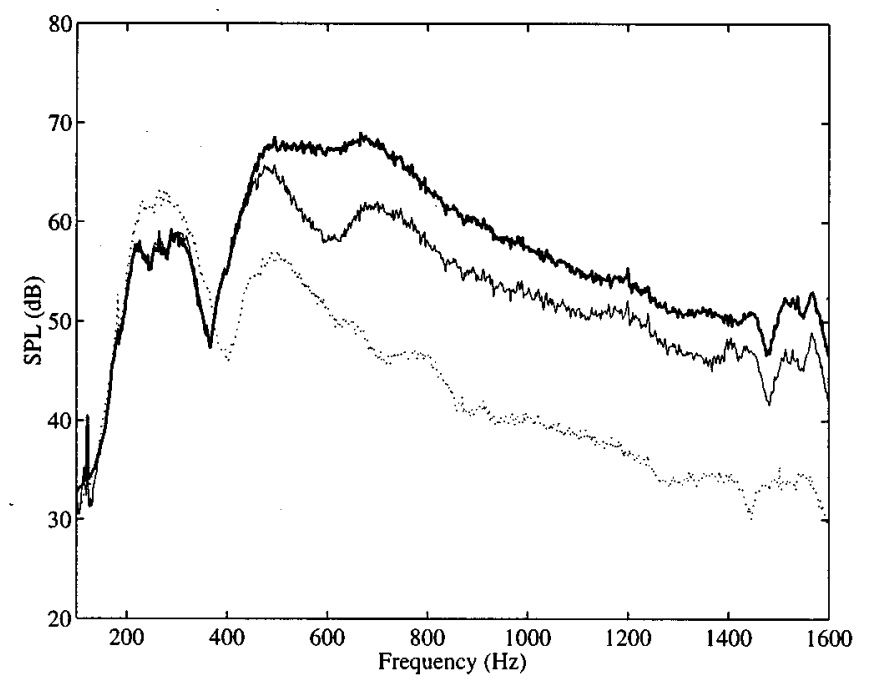

FIG. 5. Passive effects of embedded PVDF layer on global sound level: piston; - plain foam; ---- smart foam.

bare piston (noise source without sound control treatment), for the piston with a plain layer of foam (noise source with common passive sound control treatment), and for the piston with smart foam (no control signal applied to PVDF actuator). Above $350 \mathrm{~Hz}$, the active layer enhances the passive performance of the acoustic foam. Indeed, in this frequency range, the passive sound attenuation associated with the smart foam is about $15 \mathrm{~dB}$ (with respect to the untreated piston source) and that associated with the plain foam is around $6 \mathrm{~dB}$. Obviously, the combined mass of the PVDF and glue layers enhances the passive performance of the acoustic foam as it causes a portion of the energy carried by incident acoustic waves to be reflected back towards the source. In the low-frequency range (below $350 \mathrm{~Hz}$ ), the plain foam has no effect on the sound radiated by the piston. This is expected due to the longer wavelength of the incident acoustic wave relative to the foam thickness. However, the passive smart foam enhances sound radiation between 200 and $350 \mathrm{~Hz}$. This may be attributed to a modification of the radiation impedance (increase of the resistive impedance) of the system due the presence of the PVDF and glue layers within the passive smart foam. In spite of this negative effect in the low-frequency range, the smart foam yields considerable passive sound reduction over the studied frequency band.

\section{HARMONIC RADIATION PASSIVE/ACTIVE CONTROL RESULTS}

Harmonic control of piston radiation was investigated for two different excitation frequencies. Control results are shown in Fig. 6(a) and (b) for 290 and $1000 \mathrm{~Hz}$, respectively. A $290-\mathrm{Hz}$ excitation frequency corresponds to a resonant frequency of the piston shaker and lies within a frequency range where the smart foam does not provide any passive attenuation. On the other hand, the $1000-\mathrm{Hz}$ driving frequency was chosen because the smart foam provides more than $10 \mathrm{~dB}$ of passive sound attenuation in this frequency range. In general, the control results show that even if the sound radiation is
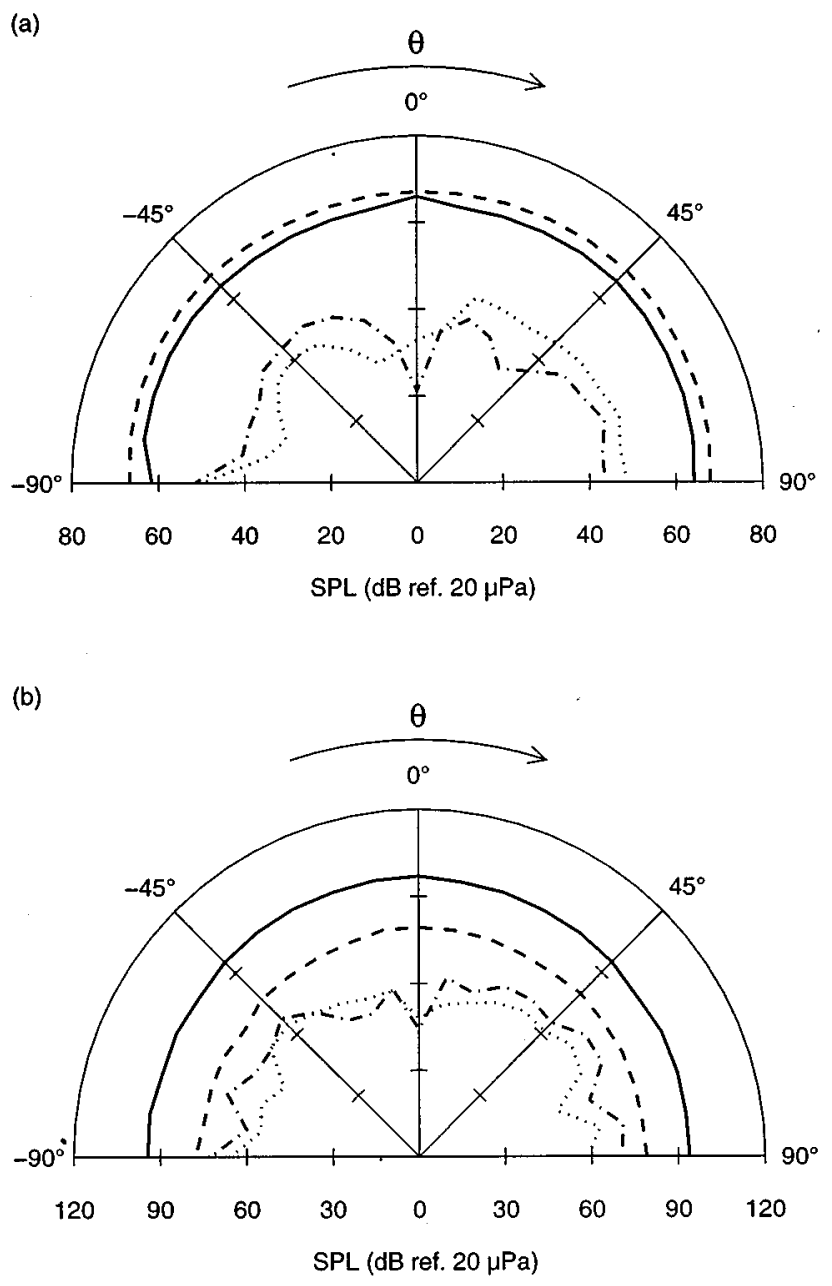

FIG. 6. Harmonic control results at (a) $290 \mathrm{~Hz}$ and (b) $1000 \mathrm{~Hz}$ : - piston; - - passive; -... passive-active series-parallel configuration; $\cdots$ passiveactive parallel configuration.

minimized at only one location in space (direction $\theta=0^{\circ}$ ), the sound attenuation under control is global. In Fig. 6(a), the global sound level is decreased by about $20 \mathrm{~dB}$ at $290 \mathrm{~Hz}$ using the series-parallel actuator. The parallel actuator yields a 16-dB global sound reduction. It is also observed that at this frequency, the passive smart foam causes a $1.5-\mathrm{dB}$ increase in the far-field radiation. The reason for this behavior has been discussed in the previous section. Figure 6(b) shows results for a $1000-\mathrm{Hz}$ excitation frequency. About $10-\mathrm{dB}$ passive sound reduction is observed while a further $10-\mathrm{dB}$ global reduction is attributed to the active PVDF actuator. The global noise cancellation was obtained as the smart foam is able to change the radiation impedance seen by the vibrating piston. The attenuation achieved by both PVDF actuator configurations is comparable at this frequency. Radiation control at other harmonic excitation frequencies was investigated and indicated that the seriesparallel actuator performed best at reducing the piston sound radiation. However, it is important to note that the control voltage required by the series-parallel configuration is greater than that needed by the parallel actuator. For example, for a $290-\mathrm{Hz}$ drive frequency, the series-parallel actuator required about 1.5 times more voltage than the parallel 
actuator to minimize the piston radiation. This observation was expected as Fig. 4 illustrated that the parallel actuator was a more efficient sound radiator than the series-parallel actuator. At $1000 \mathrm{~Hz}$, where passive sound absorption is evident, the control voltage required to drive the PVDF actuator was much lower than at $290 \mathrm{~Hz}$. Therefore, as the piston excitation frequency increases, the passive absorption provided by the smart foam increases and the voltage required to drive the PVDF actuator decreases. Applying the maximum allowable control voltage (i.e., $300 \mathrm{~V} \mathrm{rms)}$ would allow the smart foam to radiate a $60-\mathrm{dB}$ sound-pressure level at 1.5 $\mathrm{m}$ at $290 \mathrm{~Hz}$ using the parallel configuration. For the same type of excitation, a 71-dB sound-pressure level could be generated by the smart foam at $1000 \mathrm{~Hz}$. These experimental results reveal the benefits of implementing a composite active/passive system for acoustic radiation control.

\section{BROADBAND PASSIVE/ACTIVE CONTROL RESULTS}

The second control case involves broadband control of piston radiation. The piston was excited with band-limited random noise between 0 and $1600 \mathrm{~Hz}$. The error microphone was again located in the direction perpendicular to the surface of the foam $\left(1.5 \mathrm{~m}\right.$ away in direction $\left.\theta=0^{\circ}\right)$. This error microphone position represents the location of maximum directivity. The performance of the smart foam for minimizing broadband radiation at the error microphone is presented in Fig. 7(a). Below $350 \mathrm{~Hz}$, an active sound attenuation of about $10-15 \mathrm{~dB}$ is achieved for both actuator configurations. In this frequency range, it is important to note that the active component (PVDF actuator) is able to compensate for the poor performance of the passive smart foam. Above $350 \mathrm{~Hz}$, the series-parallel actuator configuration performs better than the parallel one. This is believed to be related to the nonlinear behavior associated with the parallel configuration [as seen in Fig. 4(c)]. Above $350 \mathrm{~Hz}$, about 15-dB attenuation can be attributed to the passive effect of the foam, while $10-15 \mathrm{~dB}$ attenuation is associated with the active contribution of the PVDF element for the series-parallel configuration. It was again noticed that the control signal used to drive the PVDF actuator (for all configurations) decreases in amplitude as frequency increases. Global sound levels associated with the passive/active device are presented in Fig. 7(b). It can be observed that in the low-frequency region, global active sound attenuation is achieved while using only one error microphone. For the series-parallel configuration, global active sound attenuation of about $10 \mathrm{~dB}$ is achieved up to $1200 \mathrm{~Hz}$. The smart foam modifies the resistive radiation impedance of the source yielding global noise cancellation. However, above $1300 \mathrm{~Hz}$, the amount of active reduction for both PVDF configurations seems to decrease and slight control spillover occurs. This may be attributed to the fact that the number of coefficients in the FIR filters used in the implementation of feedforward LMS controller was not sufficient to model the system response in the high-frequency range. However, even though control spillover is present, the levels never increase above that of the untreated piston. Thus, the passive sound absorption associated with the passive-active device provides some failsafe control. The (a)

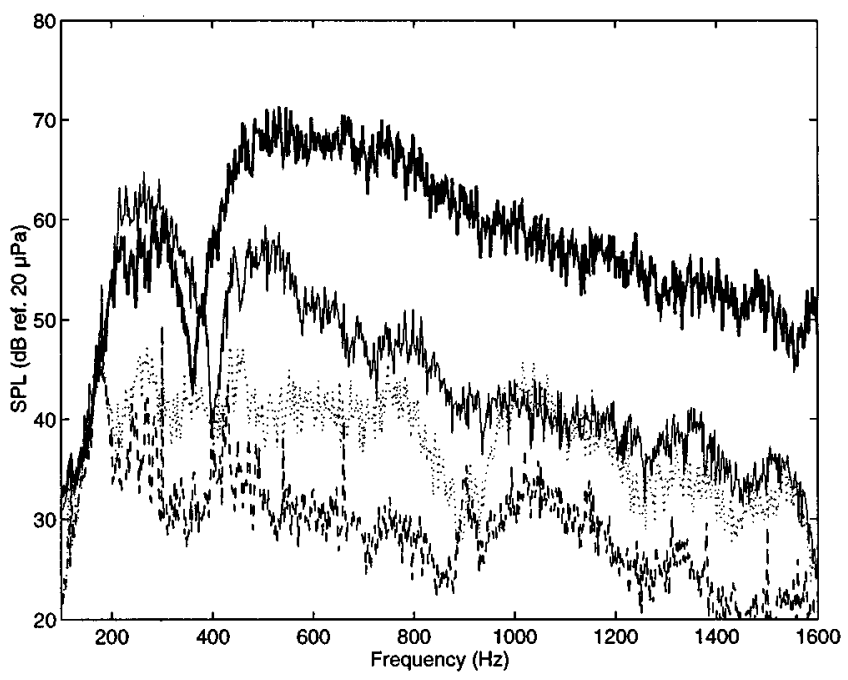

(b)

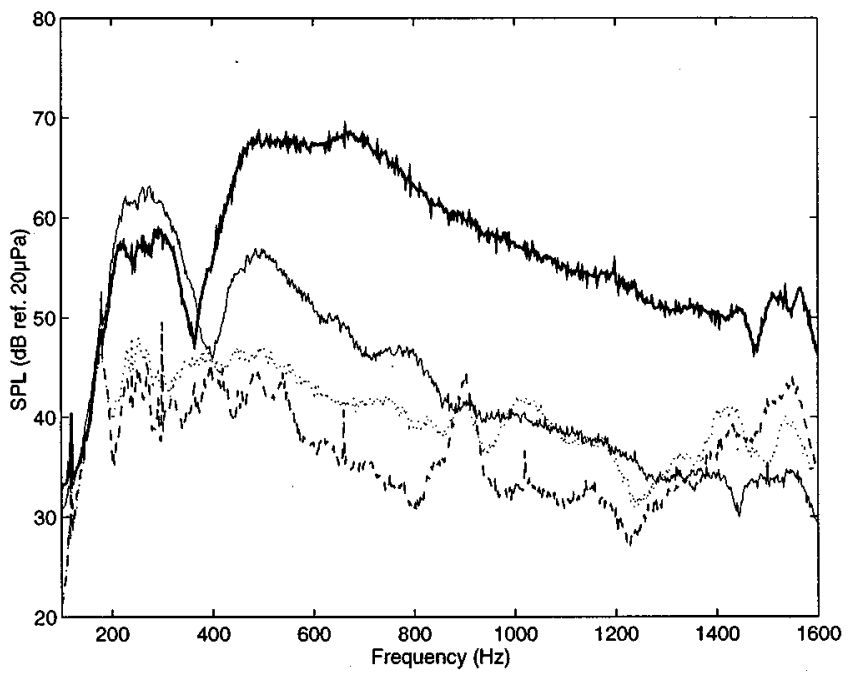

FIG. 7. Broadband control results (a) at the error microphone; (b) global sound level; - piston, - passive, ---- passive-active series-parallel configuration; $\cdots$ passive-active parallel configuration.

complimentary nature of performing active-passive control is evident as the active part controls the low-frequency range while the passive component performs best at high frequencies.

\section{CONCLUDING DISCUSSION}

In this paper, a smart foam has been introduced as a active-passive noise control device, which has many advantages over conventional noise attenuation systems. The smart foam consists of cylindrically curved sections of PVDF film embedded in partially reticulated polyurethane acoustic foam. The device is designed to reduce sound by the action of the passive absorption of the foam (effective at high frequencies) and the active input of the PVDF element driven by an oscillating electrical input (effective at low frequencies). The PVDF actuator was configured to behave in a linear sense as well as to increase its sound radiation effi 
ciency. Global cancellation of harmonic and broadband noise induced by a vibrating piston was successfully achieved by the smart foam. The passive-active device was able to modify the radiation impedance observed by the piston over a wide range of frequencies. The potential of smart foam to globally reduce low- and high-frequency sound radiating from a vibrating piston was demonstrated.

Further research will concentrate on increasing the control authority of the device (i.e., the radiation efficiency and output of the PVDF actuator). With the current configuration of smart foam, it is possible to generate a $60-\mathrm{dB}$ soundpressure level at $1.5 \mathrm{~m}$ in the low-frequency region $(\approx 300$ $\mathrm{Hz}$ ) when excited by the maximum allowable voltage. A multi-layer PVDF configuration and modification of the foam shape are two issues that are being studied to improve smart foam. The use of different error sensors (a far-field microphone is in general impractical) is also under study. The combination of completed analytical models for the porous foam and the curved PVDF film for the purpose of simulating a smart foam radiation control experiment is currently being investigated. Radiation control of more complex vibrating structures using a smart foam array will be a major area for future research.

\section{ACKNOWLEDGMENTS}

The authors gratefully acknowledge the support of this work by the Office of Naval Research under Grant No. ONR N00014-94-1-1140, the Army Research Office under Grant No. DAA H04-95-1-0037, and the NASA Langley Research Center.
${ }^{1}$ K. W. Ng, “Applications of active control,', The 1995 International Symposium on Active Control of Sound and Vibration, Active 95, Newport Beach, California, Supplemental paper, July 1995.

${ }^{2}$ M. V. Gandhi and B. S. Thompson, Smart Materials and Structures (Chapman \& Hall, London, England, 1992), p. 74.

${ }^{3}$ A. H. von Flotow, A. Beard, and Don Bailey, "Adaptive tuned vibration absorbers: tuning laws, tracking agility, sizing, and physical implementations," Proceedings of Noise-Con 94, Fort Lauderdale, Florida, May 1994, pp. 437-454.

${ }^{4}$ C. R. Fuller, J. P. Maillard, M. Mercadal, and A. H. von Flotow, 'CControl of aircraft interior noise using globally detuned vibration absorbers,' Proceedings of First Joint CEAS/AIAA Aeroacoustics Conference, Germany, Volume 1, 1995, pp. 615-624.

${ }^{5}$ Y. J. Kang, W. Tsoi, and J. S. Bolton, "The effect of mounting on the acoustical properties of finite-depth polyimide foam layers," Proceedings of Noise-Con 93, Williamsburg, Virginia, 1993, pp. 285-290.

${ }^{6}$ L. Beranek and L. Ver, Noise and Vibration Control Engineering: Principles and Applications (Wiley, New York, 1992), p. 204.

${ }^{7}$ J. S. Bolton and E. R. Green, "Smart foams for active absorption of sound,' Second Conference on Recent Advances in Active Control of Sound and Vibration, Blacksburg, Virginia, 1993, pp. 139-149.

${ }^{8}$ T. R. Howarth, V. K. Varadan, X. Boa, and V. Varadan, 'Piezocomposite coating for active underwater sound reduction,' J. Acoust. Soc. Am. 91, 823-831 (1992).

9 A. Baz, “Active Constrained Layer Damping,', Proceedings of Damping 93 Conference, San Francisco, California, February 1993.

${ }^{10}$ C. R. Fuller, M. J. Bronzel, C. A. Gentry, and D. E. Whittington, “Control of Sound Radiation/Reflection with Adaptive Foams," Proceedings of Noise-Con 94, Fort Lauderdale, Florida, May 1994, pp. 429-436.

${ }^{11}$ C. R. Fuller, S. J. Elliott, and P. A. Nelson, Active Control of Vibration (Academic, London, 1996).

${ }^{12}$ P. A. Nelson and S. J. Elliott, Active Control of Sound (Academic, London, 1992).

${ }^{13}$ Polymer Technology Inc., Technical Data Sheet: "Acoustical Products-An Overview,', published by Polymer Technology Inc., Newark, Delaware.

${ }^{14}$ G. C. Tibbets, "Transducers having piezoelectric film arranged with alternating curvatures,' U. S. Patent No. 4,056,742 (November 1977).

${ }^{15}$ L. Beranek, Acoustical Measurements (Acoustical Society of America, Cambridge, MA, 1988), pp. 674-676. 